\title{
Mice Deficient in Rap1A GTPase Display Reduced Adhesion of Hematopoietic and Mesenchymal Stromal Cells but Intact Hematopoietic Repopulation Potential
}

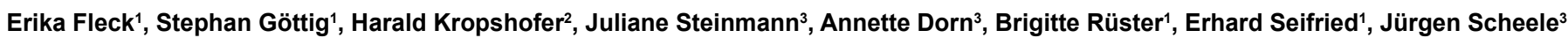
and Reinhard Henschler ${ }^{1,4 *}$

${ }^{1}$ Institute for Transfusion Medicine and Immune Hematology, German Red Cross Blood Service, Medical Center of the Johann Wolfgang Goethe University, Frankfurt (Main), Germany

${ }^{2}$ F Hoffmann-La Roche Ltd, Pharmaceuticals Division, Product Development, Basel, Switzerland

${ }^{3}$ Department of Hematology and Oncology, University Hospital Medical Center, Freiburg, Germany

${ }^{4}$ Blood Centers Zürich and Chur, Swiss Red Cross Blood Service, Zürich and Chur, Switzerland

\begin{abstract}
Background: The Guanine Triphosphatase (GTPase) Rap1A has been implicated in cell proliferation and migration in different tissues. Its role in the production, adhesion and migration of bone marrow-derived hematopoietic cells is however incompletely understood.

Methods: We analysed mice lacking one or both Rap1A alleles and measured their adhesion function and their hematopoietic repopulation capacity using competitive bone marrow transplantation in the CD45.1/CD45.2 transplant model. We further employed flow cytometry and shear stress-dependent flow chamber adhesion assays for phenotypic characterization and analysis of adhesion functions.

Results: Rap $1 \mathrm{~A}^{-/}$mice showed decreased numbers of lymphocytes and hematopoietic colony forming progenitors in the blood, while the numbers of granulocytes, monocytes or platelets were not significantly altered compared to controls. Chemokine CXCL12-induced adhesion of lineage marker depleted (Lin-) bone marrow cells to Vascular Cell Adhesion Molecule (VCAM)-1 and to Intercellular Adhesion Molecule (ICAM)-1 was significantly reduced in Rap1 $\mathrm{A}^{-1-}$ mice. However, competitive repopulation by Rap1A deficient bone marrow cells was not inferior to wild type bone marrow 9 months after transplantation. No outgrowth of Mesenchymal Stromal Cells (MSCs) was observed from bone marrow of Rap1 $\mathrm{A}^{-/-}$mice, whereas Rap $1 \mathrm{~A}^{+/}$MSCs displayed reduced expression of adhesion molecules involved in progenitor homing and impaired adhesion to primary endothelial cells and VCAM-1 under shear stress in vitro, as compared to their wild-type counterparts.
\end{abstract}

Conclusions: Rap1A regulates adhesion of immature hematopoietic cells and mesenchymal stromal cells, but appears to be redundant for hematopoietic engraftment.

Keywords: Hematopoietic stem cells; Mesenchymal stromal cells; Transplantation; Engraftment; Rap1 GTPase

\section{Introduction}

The GTPase Ras-associated protein (Rap) 1 belongs to the family of small GTPases, and has been described as a Ras antagonist in oncogenic transformed fibroblasts [1]. The two isoforms of Rap1, Rap1A and Rap1B are known to be implicated in the regulation of immune responses mainly through affecting adhesive functions and activation of lymphocyte populations [2-4]. More recently, Rap1B deficiency has been linked to defects in B cell development and angiogenesis [5-7]. Moreover, Li et al. [8] reported that neutrophilic granulocytes show a reduced oxidative burst formation in the absence of Rap1A and Jang et al. described a role for Rap1 in prostaglandin E2-induced proliferation of cord blood derived MSCs [9].

However, data are lacking which describe the function of Rap1A in undifferentiated hematopoietic cells or bone marrow-derived MSCs. We therefore investigated whether Rap1A deficient mice show alterations in levels of hematopoietic cells in blood as well as in proliferation and differentiation after bone marrow transplantation. In addition, we explored the phenotype and adhesive function of MSCs isolated from bone marrow of Rap1A deficient mice. We report here that Rap1A deficiency results in lower numbers of lymphocytes and hematopoietic colony forming progenitor cells in blood. Furthermore, Lin- enriched undifferentiated progenitor cells have a reduced capacity to adhere to VCAM-1 under shear stress after chemokine stimulation.
Likewise, Rap1A was essential for establishment of MSCs from bone marrow, Rap1A deficient MSCs displayed lower numbers of surface adhesion molecules and impaired adhesion to endothelial cells and immobilized VCAM-1 under shear stress. However, bone marrow cells from Rap $1 \mathrm{~A}^{-/}$mice displayed unperturbed engraftment potential compared with wild-type cells.

\section{Materials and Methods}

Analysis of blood cells, bone marrow cells and colony forming cells (CFCs) in mice

Animal experiments have been conducted according to the

*Corresponding author: Reinhard Henschler, MD, Ph.D., Institute for Transfusion Medicine and Immune Hematology, German Red Cross Blood Service, Medical Center of the Johann Wolfgang Goethe University, Sandhofstraße 1 D 60528 Frankfurt (Main), Germany; Blood Center Zürich SRK, Rütistrasse 19, CH-8952 Zürich-Schlieren, Switzerland, Tel: +41 58272 5117; E-mail: rhenschler@gmx.de

Received July 27, 2016; Accepted August 16, 2016; Published August 22, 2016

Citation: Fleck E, Göttig S, Kropshofer H, Steinmann J, Dorn A, et al. (2016) Mice Deficient in Rap1A GTPase Display Reduced Adhesion of Hematopoietic and Mesenchymal Stromal Cells but Intact Hematopoietic Repopulation Potential. J Stem Cell Res Ther 6: 356. doi: 10.4172/2157-7633.1000356

Copyright: ( 2016 Fleck E, et al. This is an open-access article distributed unde the terms of the Creative Commons Attribution License, which permits unrestricted use, distribution, and reproduction in any medium, provided the original author and source are credited. 
Citation: Fleck E, Göttig S, Kropshofer H, Steinmann J, Dorn A, et al. (2016) Mice Deficient in Rap1A GTPase Display Reduced Adhesion of Hematopoietic and Mesenchymal Stromal Cells but Intact Hematopoietic Repopulation Potential. J Stem Cell Res Ther 6: 356. doi: 10.4172/2157-7633.1000356

Page 2 of 8

Local Animal Welfare Commission, vote number V54 F27/07 Regierungspräsidium Darmstadt. Mice with a constitutive deletion of Rap1 A were generated and bred as previously described [3]. For analysis of blood cells, 20-50 $\mu \mathrm{l}$ peripheral blood was collected by puncture of maxillary vein of mice aged 8-14 weeks, anticoagulated with $10 \%(\mathrm{v} / \mathrm{v})$ of $0.1 \mathrm{M}$ EDTA in PBS and analyzed in an automated hemocytometer (Cell Systems, Troisdorf, Germany). Bone marrow cells were isolated for adhesion and transplantation studies from 8 to 14-week-old Rap1 $\mathrm{A}^{-1},{ }^{+/}$or wild-type mice (all CD45.2/Ly-5.2 genotype) or from wild-type CD45.1/ Ly-5.1 mice bred in our own colony. Enrichment of undifferentiated cells was performed by immunomagnetic depletion of lineage-marker expressing (Lin-) cells using the Lineage Cell Depletion kit (Miltenyi Biotec, Bergisch Gladbach, Germany) and MACS Technology according to the manufacturer's instructions. The purity of the enriched Lin- bone marrow cells was evaluated by flow cytometry using antibodies against CD11b/Mac-1, Gr-1, CD3e, CD45R/B220 and TER119 markers (Becton Dickinson, Heidelberg, Germany). For colony formation assays, isolated bone marrow cells were plated at a density of $5 \times 10^{4}$ cells $/ \mathrm{ml}$ methylcellulose (0.9\%)-containing Iscove's Modified Dulbecco's Medium (IMDM) substituted with 10\% FCS (both form Lonza, Basel, Switzerland) and $10 \mathrm{ng} / \mathrm{ml}$ each of recombinant murine Stem Cell Factor, FLT3 Ligand, Thrombopoietin, Interleukin (IL)-3, and Granulocyte-Macrophage Colony Stimulating Factor (R\&D Systems, Wiesbaden, Germany). Cells were incubated at $37^{\circ} \mathrm{C}$ in a humidified atmosphere for 14 days and scored microscopically. Expression of adhesion molecules was analyzed by flow cytometry using fluorescence-conjugated antibodies for Very Late Antigen (VLA)-4, Lymphocyte Function Antigen (LFA)1, Macrophage Antigen Complex (MAC)-1, $\alpha_{4} \beta_{7}$ integrin, CD44, or CXCR4 (Becton Dickinson).

\section{Isolation and expansion of MSCs}

Bone marrow cells were collected from femora of 8-12-week-old mice and plated at a density of $5 \times 10^{5}$ cells $/ \mathrm{cm}^{2}$ in DMEM medium supplemented with $10 \%$ FCS, $100 \mathrm{IU} / \mathrm{ml}$ penicillin, $0.1 \mathrm{mg} / \mathrm{ml}$ streptomycin and $2 \mathrm{ng} / \mathrm{ml}$ fibroblast growth factor (PAA Laboratories, Cölbe, Germany). Cells were incubated in a humidified environment at $37^{\circ} \mathrm{C}$ and $5 \% \mathrm{CO}_{2}$. Non-adherent cells were discarded after 5 days of culture and the whole medium was replaced by fresh medium. Afterwards, the medium was replaced every 3-4 days within a period of 3-4 weeks. When the layer of adherent spindle-shaped cells became sub-confluent $(70-75 \%)$, the cells were passaged by trypsin treatment, and the expression of the surface markers CD45, CD105 and CD73 was analyzed by flow-cytometry. As soon as $>98 \%$ cells displayed the phenotype $\mathrm{CD} 45{ }^{-} \mathrm{CD} 105^{+} \mathrm{CD} 73^{+}$, their differentiation potential was confirmed by successful induction of osteoplastic and adipogenic differentiation upon addition of $\mathrm{NH}$ OsteoDiff Medium or $\mathrm{NH}$ AdipoDiff Medium (Miltenyi Biotech, Bergisch-Gladbach, Germany), respectively.

\section{Competitive hematopoietic repopulation experiments}

For assessment of repopulation potential of bone marrow cells from Rap1A deficient mice, Ly5.1 recipient mice received total body irradiation with 2 doses of 5.5 Gy $24 \mathrm{~h}$ apart. One million freshly isolated total bone marrow cells from either Rap $1 \mathrm{~A}^{-/}$, Rap $1 \mathrm{~A}^{+/-}$or wildtype mice (CD45.2) were mixed and transplanted together with $1 \times 10^{6}$ bone marrow competitor cells (Ly5.1) into the pre-irradiated Ly5.1 mice by i.v. injection up to $24 \mathrm{~h}$ later. Transplanted mice recovered well from the transplant regime and were sacrificed 9 months later. Mononuclear cells from blood were isolated using red blood cell lysis buffer (Sigma, Munich, Germany) and subsequent density gradient centrifugation (Ficoll, Becton Dickinson). Blood, spleen and bone marrow cells were phenotyped by flow cytometry using CD45.1 PEconjugated and CD45.2 fluorescein isothiocyanate (FITC)-conjugated antibodies (Becton Dickinson). Donor type (CD45.2 $2^{+}$cells were gated and further analyzed for expression of CD11b/Mac-1, Gr-1, CD3e, CD45RO/B220 and TER119 markers by directly conjugated antibodies (Becton Dickinson).

\section{Parallel plate flow chamber adhesion studies}

To study interaction with recombinant adhesion ligands, parallel plate flow chambers ( $\mu$ slides, Ibidi, Munich, Germany) were pre-coated with $20 \mu \mathrm{g} / \mathrm{ml}$ (in phosphate-buffered saline (PBS)/ 1\% (v/v) bovine serum albumin (BSA) recombinant murine VCAM-1-immunoglobulin (Ig) or ICAM-1-Ig fusion proteins (Becton Dickinson) for $30 \mathrm{~min}$ at room temperature. Chambers were blocked with PBS/1\% BSA to avoid unspecific binding $30 \mathrm{~min}$ prior to the experiment. Ten minutes before the start of the experiment, chemokine CXCL12 (final concentration $1000 \mathrm{ng} / \mathrm{ml}, \mathrm{R} \& \mathrm{D}$ Systems) was immobilized on the bottom of the flow chamber. One hundred thousand Lin- cells were seeded in Hank's buffered salt solution (HBSS; Gibco, Karlsruhe, Germany) supplemented with $0.1 \%$ BSA into the flow chamber per channel and allowed to adhere for $3 \mathrm{~min}$. Then, medium flow was started with $37^{\circ} \mathrm{C}$ prewarmed $\mathrm{HBSS} / 0.1 \%$ BSA using a precision pump (Hugo Sachs Elektronik Harvard Apparatus GmbH, March, Germany) at a calculated shear stress of 0.35 dynes $/ \mathrm{cm}^{2}$. Shear stress was increased stepwise every $30 \mathrm{~s}$. Numbers of adherent cells were determined using a CCD camera and counted manually.

Interaction of MSCs with endothelial cells or VCAM-1 under shear stress. The coating of the flow chamber basement with endothelial cells was performed 1-2 days prior to the experiment. To this end, $1 \times 10^{6}$ human umbilical vein endothelial cells (HUVECs) were cultured in the flow chamber slide pre-coated with $0.1 \%$ gelatin at $37^{\circ} \mathrm{C}$ in a humidified atmosphere with 5\% $\mathrm{CO}_{2}$. Experiments were performed after overnight stimulation with $10 \mathrm{ng} / \mathrm{ml}$ Tumor Necrosis-Factor (TNF)- $\alpha$ (R\&D Systems).

To study interactions of MSCs with HUVECs at low shear stress, $1 \times 10^{6}$ wild-type or Rap1 $\mathrm{A}^{+/-}$MSCs were flushed in pre-warmed $\left(37^{\circ} \mathrm{C}\right)$ Hank's Balanced Salt Solution (HBSS) supplemented with $0.5 \%$ BSA over a confluent HUVEC monolayer at a calculated shear stress of 0.1 dynes $/ \mathrm{cm}^{2}$, and numbers of interacting cells were determined microscopically. To study firm adhesion of MSCs, $1 \times 10^{6}$ MSCs were added in $100 \mu \mathrm{l} 37^{\circ} \mathrm{C}$ pre-warmed HBSS to the chamber and allowed to adhere to HUVECs for 10 min with or without previous TNF- $\alpha$ stimulation. Subsequently, pre-warmed HBSS with $0.5 \%$ BSA was pumped over the cells under a constant shear stress of 0.1 dynes $/ \mathrm{cm}^{2}$ until non-adherent cells were flushed away. Cells were enumerated and, subsequently, the shear stress was increased to 2 dynes $/ \mathrm{cm}^{2}$. After 10 min, the remaining adherent MSCs were counted.

The adhesion to VCAM-1 was studied after coating the flow chamber for $30 \mathrm{~min}$ at ambient temperature with VCAM-1 $(30 \mu \mathrm{L} ; 20$ $\mu \mathrm{g} / \mathrm{ml})$. Unspecific binding was blocked by adding $50 \mu \mathrm{lBS} / 1 \% \mathrm{BSA}$ for $10 \mathrm{~min}$. CXCL12 was added at $1000 \mathrm{ng} / \mathrm{ml}$ in $100 \mu \mathrm{l}$ PBS/1\% BSA. Ten to the sixth MSCs were added in $100 \mu \mathrm{l}$ pre-warmed HBSS and left for $5 \mathrm{~min}$ before flow was started. Shear stress was initiated at 0.1 dynes/ $\mathrm{cm}^{2}$ for $30 \mathrm{~s}$, and then increased stepwise every 30 s reaching up to 2.5 dynes $/ \mathrm{cm}^{2}$. Numbers of adherent cells were documented at the end of the 30s period for each shear stress level. For all assays, representative pictures of each chamber were taken at each step of the experiment to assess numbers of adherent MSCs. 
Citation: Fleck E, Göttig S, Kropshofer H, Steinmann J, Dorn A, et al. (2016) Mice Deficient in Rap1A GTPase Display Reduced Adhesion of Hematopoietic and Mesenchymal Stromal Cells but Intact Hematopoietic Repopulation Potential. J Stem Cell Res Ther 6: 356. doi: 10.4172/2157-7633.1000356

Page 3 of 8

\section{Statistical analysis}

A two-tailed Student's $t$ test was performed to evaluate the significance of the results. P values $\leq 0.05$ were considered as significant.

\section{Results}

\section{Peripheral blood cell numbers in Rap1 A deficient mice}

To addressed the potential role of the GTPase Rap1A in hematopoietic cell development, we first determined the levels of mature cell types in blood. Stem cell potential was assessed by competitive hematopoietic repopulation of Rap1A deficient and wild-type bone marrow cells in pre-irradiated mice by bone marrow transplantation. In addition, we asked whether Rap1A deficient undifferentiated hematopoietic cells may be defective in adhesion functions. To confirm the genotype of the knockout mice, PCR amplification of normal and deleted Rap1 alleles with different primer pairs was used as previously published [3] (Figure 1A). Some genotypings yielded weak bands in the heterozygous animals. From a total of 97 littermates, we obtained $52(53.6 \%)$ heterozygous animals, corresponding to the expected Mendelian inheritance ratio. In contrast, we obtained only $8(8.2 \%)$ Rap1A ${ }^{-/}$mice. The numbers of neutrophils, monocytes, and platelets were not significantly different in the blood of wild-type or knockout mice (Figure 1B). However, we found a significant decrease in the number of lymphocytes compared with wild-type controls (Figure 1B). In addition, the frequency of granulocyte-macrophage myeloid CFCs dropped threefold in blood of Rap $1 \mathrm{~A}^{-/}$mice compared to controls (Figure 1C). These data indicate an influence of Rap1A on levels of lymphocytes and some hematopoietic progenitor cells in blood of the mice.

\section{Rap1A is implicated in adhesion of hematopoietic progenitors}

We next explored whether the reduced numbers of hematopoietic progenitor cells found in the blood of Rap $1 \mathrm{~A}^{-1-}$ mice were associated
A
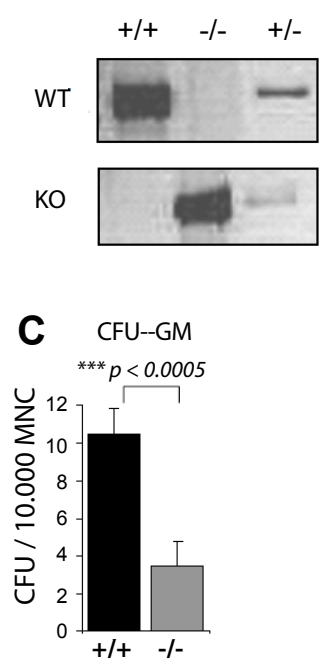

B
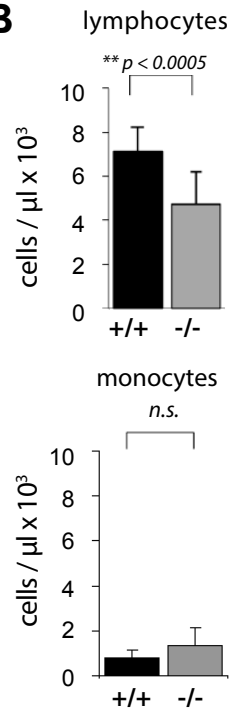

neutrophils

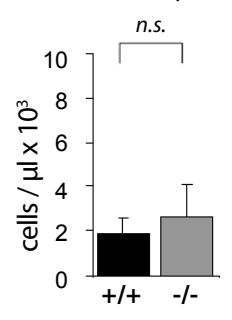

platelets

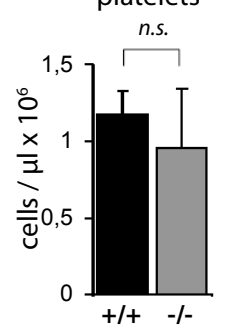

Figure 1: Genotype and peripheral blood characteristics of Rap1A deficient mice. (A) PCR genotyping result for the normal and deficient Rap1A alleles with 700 bp bands. (B,C) Cell numbers in blood of Rap1A wild-type (WT, black columns) and -/- mice (grey columns) as analyzed in an automated blood cell analyzer (B) or colony forming cell CFC assay (C). Bars represent mean of the values $\pm S D ; n=5-7$. ${ }^{* *}$ and ${ }^{* * *}$ indicate statistically significant differences.; n.s. indicates "not statistical significant". with a decreased expression of relevant adhesion molecules, mediatinge interaction of hematopoietic progenitors with endothelial cells. The integrins VLA-4, LFA-1 and Mac-1, which are ligands to VCAM1 and ICAM-1 on endothelial cells, as well as the CXCL12 receptor CXCR4 and CD44 were generally expressed at similar levels both in wild-type and Rap1A deficient Lin- cells (Figures 2A and 2B). We then analyzed the adhesion function of Lin- progenitors from bone marrow of wild-type, heterozygous or knockout mice to the integrin ligands VCAM-1 or ICAM-1 in a parallel flow chamber. Since the chemokine CXCL12 has been previously implicated in mobilization of hematopoietic progenitor cells and strengthening of integrin-mediated adhesion, we included investigation of the role of co-immobilized CXCL12 on integrin-mediated adhesion of Lin- cells. Already at low $\left(0.35\right.$ dynes $\left./ \mathrm{cm}^{2}\right)$ shear stress, and pertaining to high shear stresses of up to 15 dynes $/ \mathrm{cm}^{2}$, Rap $1 \mathrm{~A}^{-/}$Lin- cells showed a slightly decreased adhesion to ICAM-1 but not VCAM-1 compared to wild-type controls (Figure 2C). However, the CXCL12- induced increase in adhesion to both, VCAM-1 and ICAM-1 was significantly lower in Rap1A deficient Lin- cells compared to wild type controls (Figure 2C). Thus, although the integrin receptors for VCAM-1 and ICAM-1 as well as CXCR4 were expressed at comparable levels on both wild-type and knockout bone marrow cells, Rap1A deficient Lin- bone marrow cells showed a deficiency in integrin-mediated adhesion stimulated by the chemokine CXCL12.

\section{Role of Rap1A in engraftment after bone marrow transplantation}

To investigate whether this adhesion defect also translates into an engraftment defect, we transplanted freshly isolated bone marrow cells from Rap $1 \mathrm{~A}^{-/}$or Rap $1 \mathrm{~A}^{+-}$mice together with an equal number of wild-type CD45.1 competitor bone marrow cells into lethally preirradiated recipient mice. As shown in Figure 3A, the proportions of donor type hematopoietic cells were generally similar in bone marrow, blood and spleen 9 months after competitive repopulation of mice with wild-type, Rap $1 \mathrm{~A}^{+/}$or Rap $1 \mathrm{~A}^{-/}$bone marrow cells and Ly 5.1 wild-type bone marrow cells. A relatively small 22\%) increase in the proportion of donor-derived cells was detected only in the bone marrow of mice receiving Rap $1 \mathrm{~A}^{-/}$donor cells (Figure $3 \mathrm{~A}$ ); this difference was however not found in blood or spleen of the same recipient mice. Similar findings were obtained when analyzing subfractions of leukocytes in hematopoietic tissues or blood of the recipient mice (Figure 3B). Together, these data demonstrate that bone marrow cells from Rap1A deficient mice display adhesion deficits to stromal ligands, but do not harbour overt deficits in competitive hematopoietic reconstitution ability.

\section{Role of Rap1A in establishment of MSCs in culture, and} adherence of MSCs to endothelial cells and VCAM-1

We next asked whether bone-marrow derived stromal cells, which are known to be critically implied in the regulation of hematopoiesis, and which are typically represented by MSCs isolated from bone marrow, may be affected by Rap $1 \mathrm{~A}$ deficiency. To this end, whole bone marrow cells from Rap $1 \mathrm{~A}^{+/+}$, Rap $1 \mathrm{~A}^{+/}$and Rap $1 \mathrm{~A}^{-/-}$mice were depleted of hematopoietic cells by plastic adherence and cultured under conditions favoring MSC growth. While we were able to successfully isolate and expand MSCs from Rap $1 \mathrm{~A}^{+/+}$and Rap1 $1 \mathrm{~A}^{+/-}$mice, no MSC outgrowth was seen with bone marrow cells isolated from Rap1 $\mathrm{A}^{-1}$ mice (Figure 4A). Still, MSCs could be established from Rap1A ${ }^{+/-}$bone marrow. However, we observed slower growth within the initial 50 days, and an acceleration thereafter in MSC cultures derived from Rap1 $\mathrm{A}^{+/}$ 
Citation: Fleck E, Göttig S, Kropshofer H, Steinmann J, Dorn A, et al. (2016) Mice Deficient in Rap1A GTPase Display Reduced Adhesion of Hematopoietic and Mesenchymal Stromal Cells but Intact Hematopoietic Repopulation Potential. J Stem Cell Res Ther 6: 356. doi: 10.4172/2157-7633.1000356

A

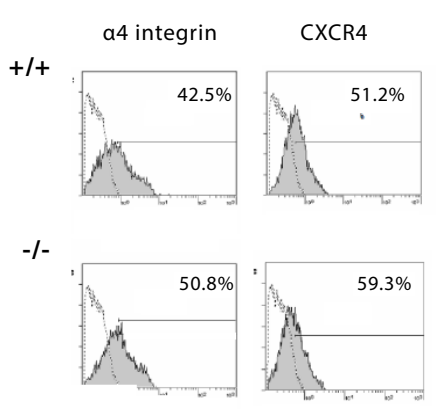

Log fluorescence
B

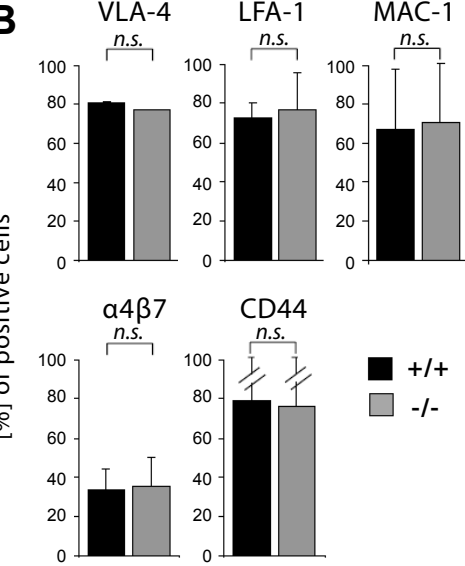

C

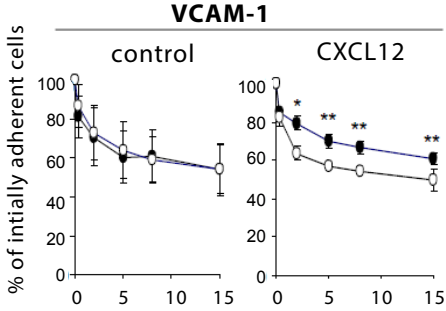

ICAM-1

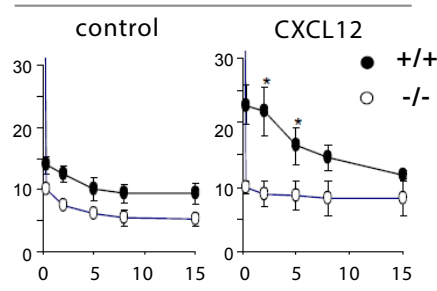

Figure 2: Expression and function of adhesion molecules in Rap1A deficient Lin- bone marrow cells. (A, B) Analysis of adhesion molecule expression. Bone marrow cells from wild-type and Rap $1 \mathrm{~A}^{-/-}$mice were assessed by flow cytometry using fluorescence-conjugated antibodies against the indicated antigens. (A) Histograms from representative determinations; (B) quantitative evaluation (means \pm SD) from wild-type (WT, black columns) and Rap1 $\mathrm{A}^{-/-}$mice (grey columns). (C) Adhesion capacity of bone marrow cells. Lin- bone marrow cells were isolated from wild-type (closed circles) and Rap $1 \mathrm{~A}^{-/-}$mice (open circles). $3 \times 10^{5}$ cells were seeded into parallel plate flow chambers pre-coated with $2 \mu \mathrm{g} / \mathrm{ml} \mathrm{VCAM-1} \mathrm{or} \mathrm{ICAM-1-lg} \mathrm{fusion} \mathrm{proteins.} \mathrm{Shear} \mathrm{flow} \mathrm{was} \mathrm{started} \mathrm{at} 0.35 \mathrm{dynes} / \mathrm{cm}^{2}$ and increased every $30 \mathrm{~s}$ using the indicated shear stresses. CXCL12 was added to the chambers for immobilization at a concentration of $800 \mu \mathrm{g} / \mathrm{ml} 10 \mathrm{~min}$ before the assays where indicated. Numbers of adherent cells were enumerated using a CCD camera and manual counting. Values are means; $n=5 .{ }^{*}$ indicates statistically significant differences.

\section{A}

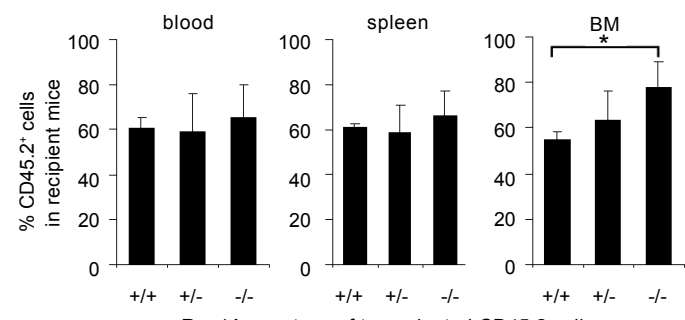

Rap1A genotype of transplanted CD45.2 cells

B

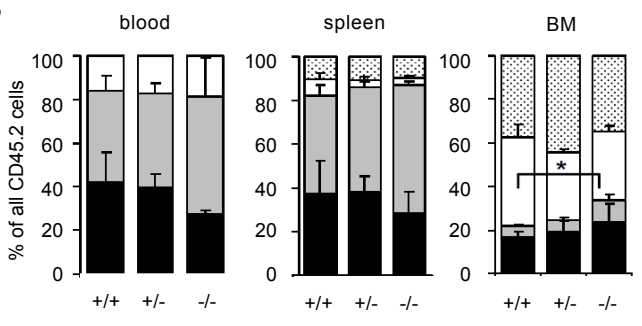

Rap1A genotype of transplanted CD45.2 cells

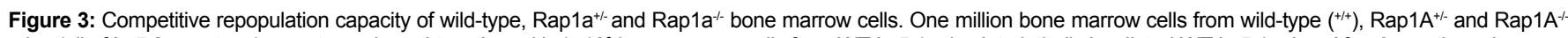
mice (all of Ly5.2 genotype) were transplanted together with $1 \times 10^{6}$ bone marrow cells from WT Ly5.1 mice into lethally irradiated WT Ly5.1 mice. After 9 months, mice were sacrificed and blood, spleen, and bone marrow cell suspensions were prepared. (A) Flow cytometric analysis of bone marrow 9 months after transplantation. Donor type repopulation was quantified by flow cytometry using CD45.1-PE labeled and CD45.2-FITC labeled antibodies and the relative percentages were calculated. Values are means $+\mathrm{SD} ; n=3-5$ per group. * indicates statistically significant difference; $p<0.05$. (B) Cells prepared from the mice in the experiment described in Figure 3 were analyzed by flow cytometry for expression CD45.2 as donor marker in subpopulations of B lymphocytes (black), T lymphocytes (grey), myeloid cells (white) or erythrocytic and other cells (dotted fields) 9 months after transplantation using anti-B220, anti-CD3, anti-Gr-1 and/or anti-Ter119 antibodies. Values represent means \pm SD; $n=3-5$ per group * indicates statistically significant difference between the sums of $B$ and $T$ lymphocytes between mice that had received wild-type or Rap $1 A^{-1-}$ donor cells; $p<0.05$. 
Citation: Fleck E, Göttig S, Kropshofer H, Steinmann J, Dorn A, et al. (2016) Mice Deficient in Rap1A GTPase Display Reduced Adhesion of Hematopoietic and Mesenchymal Stromal Cells but Intact Hematopoietic Repopulation Potential. J Stem Cell Res Ther 6: 356. doi: $10.4172 / 2157-7633.1000356$

Page 5 of 8
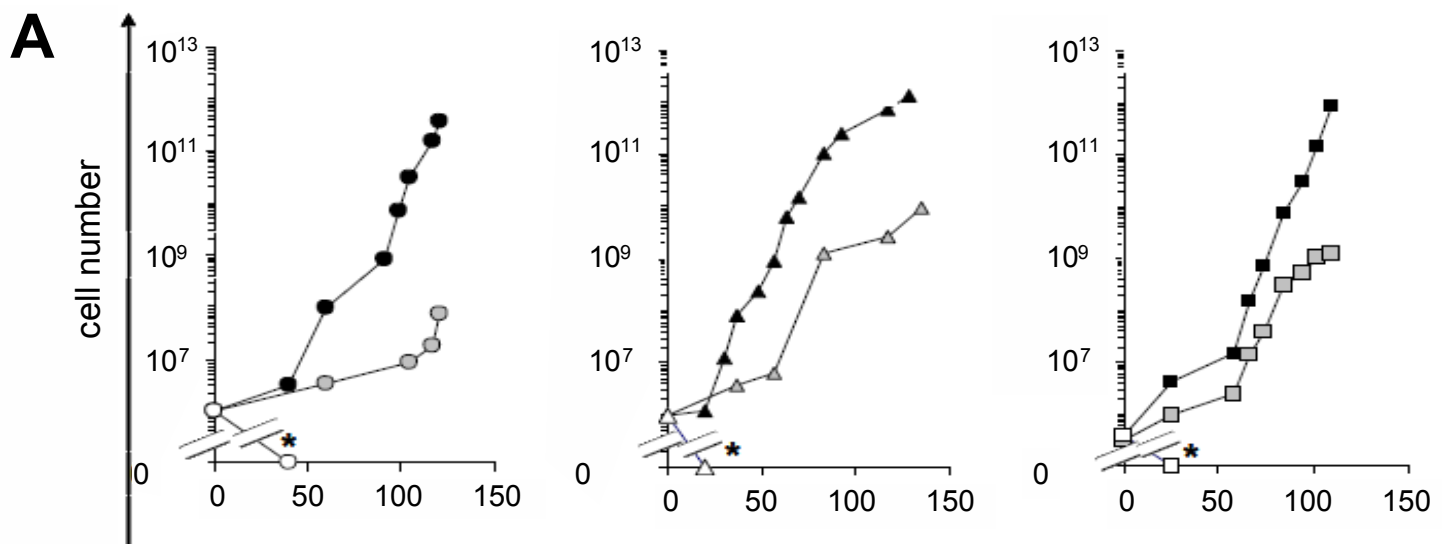

days in culture

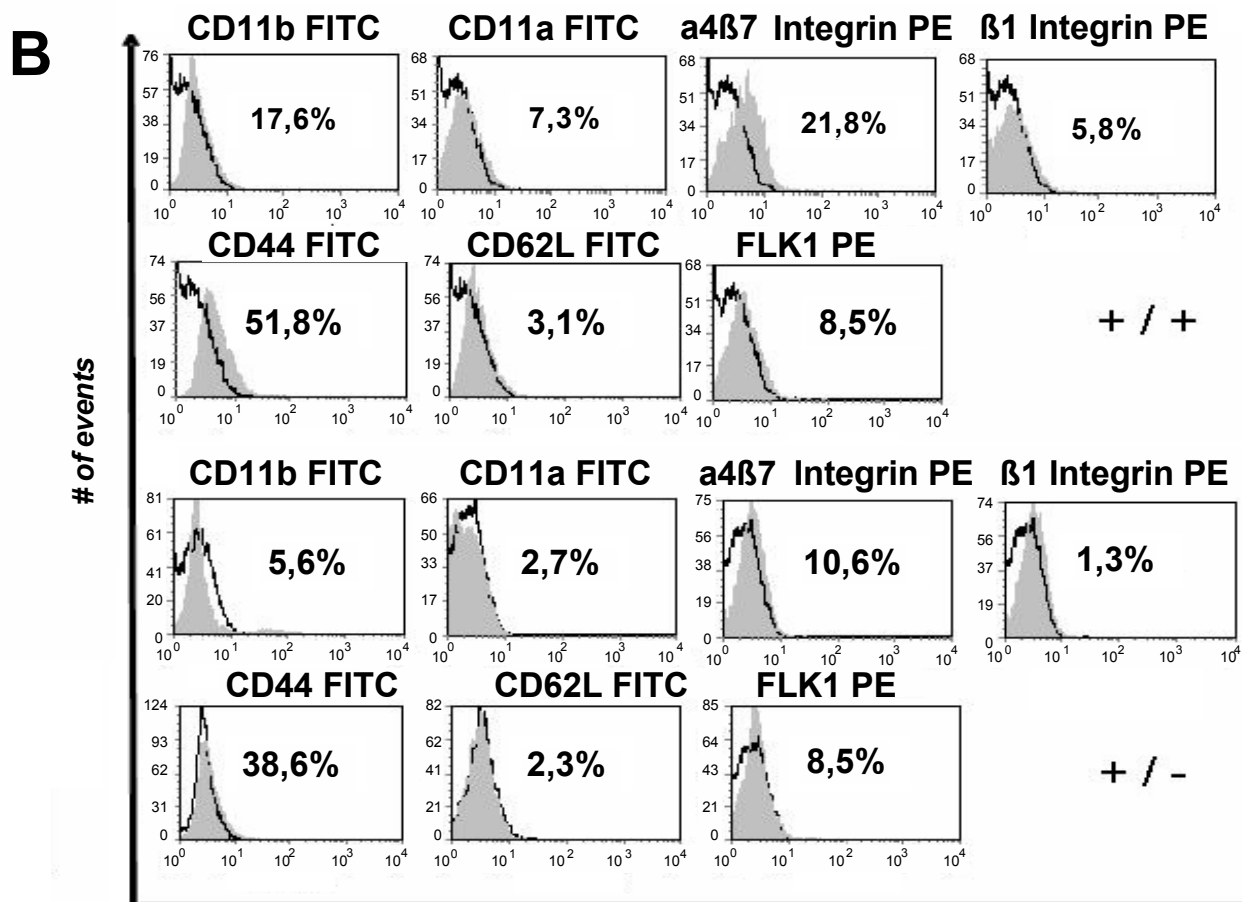

log fluorescence

Figure 4: Growth kinetics and expression of adhesion molecules of MSC isolated from wild-type, Rap1 $\mathrm{A}^{+/}$and Rap1 $\mathrm{A}^{-/-}$bone marrow cells. (A) Growth curves of three independent experiments attempting to culture MSC from three different mice are shown. Wild-type mice (filled symbols), Rap1 $\mathrm{A}^{+/-}$mice (grey symbols) and Rap1 $\mathrm{A}^{-/-}$ mice (open symbols). *Cultures were terminated because of lack of live cells. (B) Flow-cytometry of adhesion molecules expressed on the surface of MSC. MSC were stained with monoclonal antibodies directed against the indicated antigens (filled areas) or isotype control antibodies (open areas). Inserted values represent percentpositive cells. Wild-type MSC (+/+) and Rap1 $\mathrm{A}^{+/} \mathrm{MSC}(+/-)$ were analyzed. A representative result of three experiments is shown.

mice compared to wild-type mice (Figure 4A). These data implicate an adhesion defect of MSCs lacking Rap1A. Immunophenotyping using flow-cytometry revealed that the MSC cultures were positive for MSC-specific surface markers, such as CD73, CD105 or CD13, without notable differences between wild-type and Rap1A ${ }^{+/-}$MSCs (data not shown). Furthermore, wild-type and Rap $1 \mathrm{~A}^{+/}$MSC were equally able to differentiate into adipogenic or osteogenic cells (data not shown).

In search for an underlying cause of the adhesion deficits in Rap1A deficient MSC, we next examined the expression of several adhesion molecules on wild-type and Rap1 $\mathrm{A}^{+/-}$MSCs. We found a slightly lower expression of adhesion molecules CD11a, CD11b, $\alpha_{4} \beta_{7}$ integrin, $\beta_{1}$ integrin, CD44 and CD62L on Rap1 $\mathrm{A}^{+/-}$MSC compared to MSC from wild-type mice by flow-cytometry (Figure 4B).

To examine whether the differential expression of adhesion molecules translates into differential interaction with endothelial cells, we tested the interaction of MSCs with immobilized HUVEC cells in the parallel plate flow chamber model. In this model, MSCs were studied for their adhesion capacity on TNF- $\alpha$-pre-stimulated or unstimulated HUVEC at a relatively low shear shear stress of 0.1 dynes/ $\mathrm{cm}^{2}$ or 2 dynes $/ \mathrm{cm}^{2}$, to mimic the conditions found in post-capillary venules. We found a trend, although no statistical significance, towards a lower number of Rap1 $\mathrm{A}^{+/-}$MSCs interacting with HUVECs 
Citation: Fleck E, Göttig S, Kropshofer H, Steinmann J, Dorn A, et al. (2016) Mice Deficient in Rap1A GTPase Display Reduced Adhesion of Hematopoietic and Mesenchymal Stromal Cells but Intact Hematopoietic Repopulation Potential. J Stem Cell Res Ther 6: 356. doi: 10.4172/2157-7633.1000356

Page 6 of 8

in comparison with wild-type MSCs, independent of whether the HUVEC layer was stimulated with TNF-a or not (Figure 5A). We next analysed the ability of MSCs to firmly adhere to HUVECs (Figure 5B). Whereas no significant differences were detected in unstimulated HUVECs, the adhesion capacity of wild-type MSC was significantly higher compared with Rap1 $\mathrm{A}^{+/-}$MSC after pre-stimulation of HUVEC with TNF- $\alpha$ (Figure 5B). Finally, we analysed the adhesion capacity of MSC to immobilized recombinant VCAM-1 (Figure 5C). Rap1 $\mathrm{A}^{+1-}$ MSCs were inferior to wild-type MSCs in adhesion to VCAM-1, both in the absence and presence of the stimulator CXCL12. Together, these data demonstrate a role of Rap1A in adhesion to plastic during the outgrowth phase from bone marrow, and in firm adhesion of MSCs via the integrin VLA-4 - VCAM axis and co-stimulated through CXCL12.

\section{Discussion}

So far, limited data are available describing the function of Rap1A after genetic ablation in hematopoietic cells. The complete ablation of the Rap1B gene has been shown to lead to embryonic lethality, due to developmental abnormalities during early vasculogenesis, pointing out an important role of Rap1B in mesodermal development $[3,5]$. It is not clear whether such defects would also contribute to the lower birth rate in the Rap1A deficient mice which we found. Yan et al. [10] have recently reported that a knockdown of either Rap1A or B in endothelial cells by siRNA leads to a loss of vascular permeability and endothelial tube formation. In previous collaborative work with Chavakis et al. [6] we have shown that Rap1A or Rap1B deficiency is associated with a reduction of integrin activation in endothelial cells, using both siRNA mediated knockdown of Rap1 A and B, and the Rap1A knockout mouse model. In view of the role of Rap1 A and B in endothelial cell function, it was of interest to follow potential defects in hematopoietic cells with regard to adhesion to stromal ligands and hematopoietic engraftment, which are both also known to be regulated by integrins.

Our findings on blood cell numbers of myeloid hematopoietic cells are comparable to our previous results analysing Rap1A deficient $\mathrm{T}$ lymphocytes, where we observed a reduction in numbers of circulating T lymphocytes [3]. In addition to the adhesion defect of T lymphocytes on immobilized integrin ligands described earlier [3], we here report a significant adhesion defect to immobilized integrin ligands also in immature myeloid cells of the lin- fraction. So far, an adhesion deficit of hematopoietic cells has only been reported in the human leukemic hematopoietic cell line UT-7 after overexpression of RapGAP1, resulting in downregulation of Rap1 activity [11,12]. Our data with Rap1A deletion in primary cells demonstrates the direct involvement of Rap1A in integrin activation in the progenitors. In addition, we show that primarily the CXCL12-induced adhesion is affected. Other than

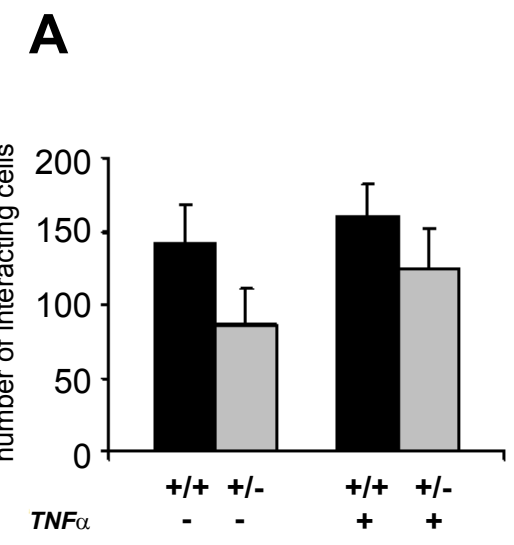

B

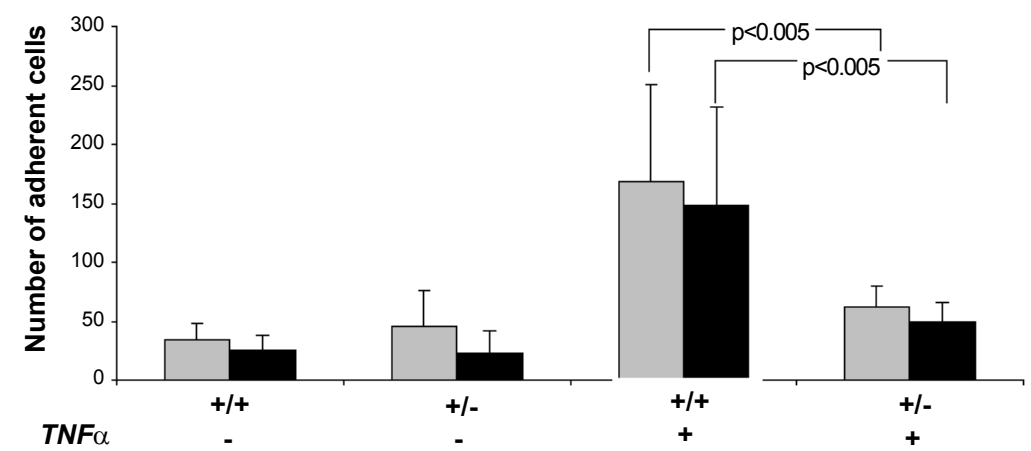

Figure 5: Adhesion of MSC on endothelial cells under shear flow. (A) Interaction of flowing MSC with HUVEC. Rap1 $\mathrm{A}^{+/+}$and Rap1 $\mathrm{A}^{+/-}$MSC were flushed at a calculated shear stress of 0.1 dynes $/ \mathrm{cm}^{2}$ over a HUVEC layer in the absence and presence of TNF- $\alpha$ in a parallel flow chamber, and numbers of interacting cells were determined as described in materials and methods. Values are means $\pm S D ; n=3$; no statistical differences were noted between test and controls. (B) Adhesion of MSC on HUVEC. MSC from Rap $1 \mathrm{~A}^{+/+}$and Rap1 $\mathrm{A}^{+/}$mice were seeded on HUVEC monolayer in a parallel plate flow chamber and analyzed after application of a calculated shear stress of 0.1 dynes $/ \mathrm{cm}^{2}$ (grey bars) or 2 dynes $/ \mathrm{cm}^{2}$ (black bars) as described in materials and methods. Values are means $\pm S D ; n=3$. (C) Adhesion of MSC on immobilized VCAM-1. MSC from Rap1 $\mathrm{A}^{+/+}$and Rap1 $\mathrm{A}^{+/-}$mice were allowed to settle for $5 \mathrm{~min}$ in a flow chamber pre-coated with recombinant VCAM-1 before the indicated shear stress was applied for $30 \mathrm{~s}$ each. In parallel, CXCL12 was pre-coated as indicated. The numbers of adherent cells in four fields were counted. Values are means \pm SD of a representative experiment. 
Citation: Fleck E, Göttig S, Kropshofer H, Steinmann J, Dorn A, et al. (2016) Mice Deficient in Rap1A GTPase Display Reduced Adhesion of Hematopoietic and Mesenchymal Stromal Cells but Intact Hematopoietic Repopulation Potential. J Stem Cell Res Ther 6: 356. doi: 10.4172/2157-7633.1000356

Page 7 of 8

previous studies, we have employed defined shear stress conditions. Other authors, using Rap1A knockout mice, have reported a migration defect of splenic $\mathrm{B}$ and $\mathrm{T}$ lymphocytes in transwell migration assays and in adhesion assays involving the vitronectin and fibronectin analogue CH-296 [8]. They also analyzed transwell migration of LinSca1+ hematopoietic progenitor cells from bone marrow, and found their migration suppressed in the absence of Rap1A. Ghandour et al. [13], found no significant role of the Rap1 activator CalDAG-GEFI in VCAM-1-mediated adhesion in hematopoietic cells. Our results showing adhesion defects in the absence of Rap1 indicate that other Rap1 activators are likely important for integrin activation. Our studies are in concordance with previous results by Shimonaka et al. in mature hematopoietic cells using dominant negative Rap1 constructs and cells with a knockout of the Rap1GAP Spal [14]. Satyanarayana et al. [15] in a model with genetic ablation of RapGEF2 show that this Rap1 activator is dispensable during adult hematopoiesis, but that embryonic hematopietic cells displayed defects in proliferation and migration, as well as in vitro colony formation ability. Carmona et al. [16] demonstrate that in human endothelial progenitor cells, EPAC1 activation is mediating integrin activation. Molina-Ortiz et al. [17] using genetic ablation of the Rap1 modulator Rasa3 show a role of this type of Rap1 modulation in controlling integrin signaling in megakaryocytes and their differentiation into proplatelets.

A phenotype of Rap1B deficiency in B cell development was reported by Chen et al. [7], who described delayed development of B cells resulting in altered $\mathrm{B}$ and $\mathrm{T}$ lymphocyte homeostasis in the absence of Rap1B. Awasthi et al. confirmed the finding of a hematopoietic maturation defect of $\mathrm{B}$ and NK cells in the absence of Rap1B, implying a role mostly for Rap $1 \mathrm{~B}$ in functional activation and in homing behaviour of NK cells, and a role for the Rac activation regulator IQGAP in this process [12]. Our studies, both in untransplanted Rap $1 \mathrm{~A}^{-/-}$mice and in chimeric mice lacking Rap1A in hematopoietic cells after bone marrow transplantation, do not indicate a major role of Rap1A in $\mathrm{B}$ cell maturation. However, a compensation of Rap1A deficiency by increased expression of endogenous Rap1B cannot be excluded also in our model. Yet, we could not analyse Rap1A or B activation separately due to lack of appropriate antibodies.

Since MSCs which have been found to support hematopoiesis show themselves a distinct adhesion and migration behaviour [18], we analysed to what extent Rap1A influences adhesion of bone marrowderived MSCs. We found a reduction by $20-80 \%$ of surface integrins on MSCs from Rap1A ${ }^{+/-}$mice compared to their wild-type counterparts (Figure 4). Consistent with this finding, Rap1A ${ }^{+/-}$MSCs were inferior to wild-type MSC in the flow chamber model under shear stress, since their adhesion to TNFa-stimulated inflammatory HUVEC cells and also to immobilized VCAM-1 was impaired (Figure 5). These findings may also explain why MSCs carrying the homozygous deletion of Rap1A were unable to survive under our cell culture conditions. This study, to our knowledge, is the first to study MSCs with genetic ablation of Rap1A. Thankamony and Sackstein [19] have used a Rap1 inhibitor and found a decreased adherence of human MSCs under flow. siRNA mediated inhibition of Rap1A was studied by Jang et al. [9], who found a decreased AKT phosphorylation in MSCs that was associated with a lower proliferation rate under Rap1 inhibition, and by Chen et al. [20] in placental derived MSCs, also after siRNA mediated Rap1 inhibition. In contrast to these studies which employ siRNA or chemical inhibition, both of which cannot $100 \%$ selectively target the Rap1A gene alone, our study using genetic ablation shows that Rap1A is indispensable for proliferation initiation of MSCs. A phenotype of defective activation of integrins in Rap1A knockout mice has been described also in endothelial cells [16] and in T cells, resulting in reduced contact dermatitis [21].

Histone methyltransferase G9a is essential for the T cell differention during murine intestinal inflammation [22]. A recent observation has shown that G9a is also involved in $\mathrm{T}$ cell adhesion via interaction with lamin B1 [23]. The impairment of adhesion in the Rap1A deficient immature hematopoietic cells and mesenchymal stromal cells might directly caused by prevention of the function of G9a. Since G9a plays a major role in the establishment and maintenance of $\mathrm{H} 3 \mathrm{~K} 9 \mathrm{me} 2$ [24] and also associates with maintenance of DNA methylation [25], it is interesting to investigate the patterns of $\mathrm{H} 3 \mathrm{~K} 9 \mathrm{me} 2$ and DNA methylatIon in the Rap1A deficient HSCs/MSCs.

Taken together, our data show a redundant role of Rap1A in the homeostasis of mature lymphocytes, myeloid cells and hematopoietic repopulating cells, but a significant role in the proliferation and adhesion in MSCs/stromal cells. The complete role of both Rap1 allelic products in these processes may be determined as soon as inducible double knockout mouse models become available, e.g., a partial knockdown of both Rap1 alleles or by breeding one or two floxed Rap1B alleles into Rap1A deficient mice and subsequently inducing hematopoietic-specific deletion. This will further clarify the role of this important regulator of integrin activation in hematopoietic and mesenchymal stem cell biology. Moreover, Rap1A may serve as a molecular target for hematopoietic and mesenchymal cell adhesion and/or mobilization processes.

\section{Authors' Contributions}

EF and SG carried out the mouse breeding and genetic analysis, EF and BR performed mouse experimentation and transplantation experiments, cell isolation, cell culture, and the flow cytometric analysis. $\mathrm{RH}$, JS and ES conceived the study, and participated in its design and coordination. EF, HK, JS and AD drafted the manuscript. All authors read and approved the final manuscript.

\section{Acknowledgement}

We are indebted to Victoria Lang and Sabrina Boehme for excellent technical support. The project was funded by the Deutsche Forschungsgemeinschaft through grants Sche 354/8-1 and He 1708/3-1.

\section{References}

1. Kitayama H, Matsuzaki T, Ikawa $Y$, Noda M (1989) A ras-related gene with transformation suppressor activity. Cell 56: 77-84. [PubMed]

2. Sebzda E, Bracke M, Tugal T (2002) Rap1A positively regulates T cells via integrin activation rather than inhibiting lymphocyte signaling. Nat Immunol 3: 251-258. [PubMed]

3. Duchniewicz M, Zwartkruis T, Kolanczyk M (2006) Rap1A deficient T and B cells show impaired integrin-mediated cell adhesion. Mol Cell Biol 26: 643-653. [PubMed]

4. Minato N, Katagiri K, Hattori M (2007) Regulation of immune responses and hematopoiesis by the Rap1 signal. Adv Immunol 93: 229-264. [PubMed]

5. Chrzanowska-Wodnicka M, Gale D, White GC, Vansluys J (2008) Defective angiogenesis, endothelial migration, proliferation, and MAPK signaling in Rap1b-deficient mice. Blood 111: 2647-2656. [PubMed]

6. Carmona G, Göttig S, Orlandi A, Scheele J, Bäuerle T, et al. (2009) Role of the small GTPase Rap1 for integrin activity regulation in endothelial cells and angiogenesis. Blood 113: 488-497. [PubMed]

7. Chen Y, Yu M, Podd A, Wen R, Chrzanowska-Wodnicka M, et al. (2008) A critical role of Rap1b in B-cell trafficking and marginal zone B-cell development. Blood 111:4627-4463. [PubMed]

8. Li Y, Yan J, De P, Chang HC, Yamauchi A, et al. (2007) Rap1a null mice have altered myeloid cell functions suggesting distinct roles for the closely related Rap1a and 1b proteins. J Immunol 179: 8322-8331. [PubMed]

9. Jang MW, Yun SP, Park JH, Ryu JM, Lee JH, et al. (2012) Cooperation of Epac1/Rap1/Akt and PKA in prostaglandin E2-induced proliferation of human 
Citation: Fleck E, Göttig S, Kropshofer H, Steinmann J, Dorn A, et al. (2016) Mice Deficient in Rap1A GTPase Display Reduced Adhesion of Hematopoietic and Mesenchymal Stromal Cells but Intact Hematopoietic Repopulation Potential. J Stem Cell Res Ther 6: 356. doi: 10.4172/2157-7633.1000356

Page 8 of 8

umbilical cord blood derived mesenchymal stem cells: involvement of c-Myc and VEGF expression. J Cell Physiol 227: 3756-3767. [PubMed]

10. Yan J, Ingram DA, Qilliam LA (2008) Rap1a is a key regulator of fibroblast growth factor-2 induced angiogenesis and together with Rap1b controls human endothelial cell function. Mol Cell Biol 28: 5803-5830. [PubMed]

11. Hitchcock IS, Kaushansky K (2007) Thrombopoietin promotes beta1-integrinmediated adhesion in hematopoietic cells via the small GTPase Rapl. Exp Hematol 35:793-801. [PubMed]

12. Awasthi A, Samarakoon A, Chu H, Kamalakannan R, Quilliam LA, et al. (2010) Rap1b facilitates NK cell functions via IQGAP1-mediated signalosomes. J Exp Med 207: 1923-1938. [PubMed]

13. Ghandour H, Cullere X, Alvarez A, Luscinskas FW, Mayadas TN (2007) CalDAG-GEFI in LFA-1 but not VLA-4 integrin-mediated human T-cell adhesion. Blood 110: 3682-3690. [PubMed]

14. Shimonaka M, Nakayama T, Fujita N, Katagiri K, Tsuruo T, et al. (2003) Rap1 translates chemokine signals to integrin activation, cell polarization, and motility across vascular endothelium under flow. J Cell Biol 161: 417-427. [PubMed]

15. Satyanarayana A, Gudmundsson KO, Chen X, Coppola V, Tessarollo L, et al. (2010) RapGEF2 is essential for embryonic hematopoiesis but dispensable for adult hematopoiesis. Blood 116: 2921-2931. [PubMed]

16. Carmona G, Chavakis E, Koehl U, Zeiher AM, Dimmeler S (2008) Activation of Epac stimulates integrin-dependent homing of progenitor cells. Blood 111: 2640-2446. [PubMed]

17. Molina-Ortiz P, Polizzi S, Ramery E, Gayral S, Delierneux C, et al. (2014) Rasa3 controls megakaryocyte Rap1 activation, integrin signaling and differentiation into proplatelet. PLoS Genet 10: e1004420. [PubMed]
18. Karp JM, Leng Teo GS (2009) Mesenchymal stem cell homing: The devil is in the details. Cell Stem Cell 4: 206-216. [PubMed]

19. Thankamony SP, Sackstein R (2011) Enforced hematopoietic cell E- and L-selectin ligand (HCELL) expression primes transendothelial migration of human mesenchymal stem cells. Proc Natl Acad Sci U S A 108: 2258-2263. [PubMed]

20. Chen CP, Huang JP, Chu TY, Aplin JD, Chen CY, et al. (2013) Human placenta multipotent mesenchymal stromal cells modulate trophoblast migration via Rap1 activation. Placenta 34: 913-923. [PubMed]

21. Dorn A, Zoellner A, Follo M, Martin S, Weber F, et al. (2012) Rap1a deficiency modifies cytokine responses and MAPK-signaling in vitro and impairs the in vivo inflammatory response. Cell Immunol 276: 187-195. [PubMed]

22. Antignano F, Burrows K, Hughes MR, Han JM, Kron KJ, et al. (2014) Methyltransferase G9A regulates $T$ cell differentiation during murine intestinal inflammation. J Clin Invest 124: 1945-1955. [PubMed]

23. Zhang X, Cook PC, Zindy E, Williams CJ, Jowitt TA, et al. (2016) Integrin a4ß1 controls G9a activity that regulates epigenetic changes and nuclear properties required for lymphocyte migration. Nucleic Acids Res. 44: 3031-3044. [PubMed]

24. Tachibana M, Sugimoto K, Nozaki M, Ueda J, Ohta T, et al. (2002) G9a histone methyltransferase plays a dominant role in euchromatic histone $\mathrm{H} 3$ lysine 9 methylation and is essential for early embryogenesis. Genes Dev 16: 17791791. [PubMed]

25. Zhang T, Termanis A, Özkan B, Bao XX, Culley J, et al. (2016) G9a/GLP Complex Maintains Imprinted DNA Methylation in Embryonic Stem Cells. Cell Rep 15: 77-85. [PubMed] 